\title{
Reduced CAMP/CREB signaling in the dentate gyrus is involved in facilitation of fear extinction in the MPTP-induced mouse model of Parkinson's disease
}

\author{
Toshiaki Ishii, Ken-Ichi Kinoshita, Yoshikage Muroi
}

Department of Basic Veterinary Medicine, Obihiro University of Agriculture and Veterinary Medicine, Japan

Parkinson's disease (PD) is a neurodegenerative disorder characterized by progressive loss of dopaminergic (DAergic) neurons in the substantia nigra pars compacta and reduction of DAergic fibers in the striatum, resulting in extrapyramidal motor dysfunctions such as tremor, rigidity, and bradykinesia. In addition, cognitive impairment, which sometimes appears before the onset of motor dysfunction, appear in about $40 \%$ of PD patients. However, the etiology of the cognitive impairment associated with PD is not well understood. Recently, we reported that PD mice produced by 1methyl-4-phenyl-1,2,3,6-tetrahydropyridine (MPTP) show facilitation of hippocampal memory extinction in the contextual fear conditioning test, which may be the cause of cognitive impairment in PD. To investigate the mechanism of facilitation, we examined the cAMP/CREB signaling cascade in the hippocampus. Decreased levels of cAMP and phosphorylated CREB were observed in the dentate gyrus (DG) of PD mice after an extinction learning task. Moreover, systemic administration of a phosphodiesterase IV inhibitor, rolipram, improved the memory deficits with concomitant recovery of phosphorylated CREB levels in the DG. These results suggest that reduced cAMP/CREB signaling in the DG of the hippocampus leads to cognitive impairment in PD mice. 